\title{
Análisis de Modelos Cinéticos de Polimerización vía Simulación Matemática
}

\author{
Pastor Rivero $^{1}$ y Rafael Herrera ${ }^{2}$ \\ (1) Universidad Nacional Autónoma de México, Facultad de Estudios Superiores Cuautitlán, \\ Departamento de Ingeniería y Tecnología, Av. Primero de mayo, 54740 Cuautitlán Izcalli - México \\ (e-mail: priveromtz@msn.com). \\ (2) Universidad Nacional Autónoma de México, Facultad de Química, Departamento de Ingeniería \\ Química, Edificio E, Ciudad Universitaria, 04360 México, D.F. - México
}

\begin{abstract}
Resumen
El objetivo de este trabajo es plantear el uso de la simulación matemática en sistemas de polimerización para obtener un mayor conocimiento sobre la cinética de la reacción. Para este fin, se presentan dos ejemplos ilustrativos, en los cuales, mediante un análisis por simulación se ha podido establecer o aclarar algún aspecto del mecanismo de reacción. En el primer ejemplo se aborda el problema de construir un modelo. Se utiliza un caso de copolimerización de monómeros que forman complejos del tipo donador aceptador de electrones. De los resultados del análisis de sistemas de este tipo, fue posible construir un modelo de terpolimerización. En el segundo ejemplo, se describe el planteamiento de un modelo de cinética de polimerización aniónica de estireno iniciada con alquilos de litio. A partir del análisis por simulación se establece la hipótesis de una participación de los agregados cruzados de iniciador-polímero en las reacciones de iniciación. Este nuevo esquema cinético permite explicar la aceleración de la reacción de iniciación y la evolución de pesos moleculares promedio del polímero.
\end{abstract}

\section{Analysis of Polymerization Kinetic Models through Mathematical Simulation}

\begin{abstract}
This work aims to explain the usefulness of mathematical simulation of polymerization systems for increasing our insight on their kinetics. For illustrative purposes, two examples are described where some topic of the reaction mechanism has been established or clarified by means of an analysis by simulation. The first example is on the model development problem. The kinetics of copolymerization of monomers that form electron donor-acceptor complexes is considered. From the analysis of this kind of systems, it was possible to develop a terpolymerization model. In a second example, it is outlined a kinetic model for the anionic polymerization of styrene initiated by alkyllithium. From the analysis by simulation of this polymerization system, the hypotheses of participation of initiatorpolymer cross aggregates in initiation reactions is established. This novel kinetic scheme allows explaining the acceleration of initiation reaction and the evolution of polymer molecular weight averages.
\end{abstract}

Keywords: polymerization kinetics, simulation, anionic polymerization, alkyllithium aggregates 


\section{INTRODUCCIÓN}

Un modelo matemático de cinética de polimerización, es una manera de sistematizar el conocimiento que se tiene del mecanismo de reacción. También es una manera de aclarar y destacar lo que se desconoce del mecanismo. La importancia de desarrollar y mejorar modelos de cinética de polimerización, radica en la capacidad de poder predecir, y por lo tanto controlar, la velocidad de reacción y la estructura molecular del polímero formado, aspectos básicos en el diseño y operación de reactores de polimerización. Más aun, el disponer de un modelo que relacione las condiciones de reacción con la estructura molecular del polímero, posee un valor práctico en razón del efecto que esta estructura tiene en las propiedades finales del material.

En un sentido general, un modelo describe la relación entre variables. Esta relación es un proceso que conecta las variables de entrada con las variables de salida utilizando una serie de parámetros. En el caso de modelos de cinética de polimerización, las variables de entrada son las condiciones de reacción: temperatura, presión, concentración de monómeros, catalizadores, iniciadores, agentes de transferencia y cualquier otro componente que se encuentre en el medio de reacción. Las variables de salida pueden ser simples, por ejemplo: velocidad de polimerización, conversión, composición de copolímeros, peso molecular promedio, etc., o pueden ser distribuciones, por ejemplo: distribución de peso molecular, distribución de longitudes de secuencia, distribución de composición, distribución de ciertas estructuras como díadas, tríadas, grupos funcionales, estructuras isotácticas o sindiotácticas, etc. Los parámetros del modelo son los coeficientes de velocidad de cada etapa de reacción, las constantes de equilibrio y cualquier otra constante del modelo.

Por otro lado, la simulación de reacciones de polimerización describe, mediante un modelo, como se desarrolla la polimerización. Es una representación aproximada del comportamiento del sistema real. En la literatura se ha tratado extensivamente el problema del modelado y simulación de diferentes sistemas de polimerización. Algunos trabajos de revisión de carácter general que resumen el estado que guarda el tema son: Dubé et al. (1997), Gao y Penlidis (1996; 2000; 2002), Achilias (2007), Krallis y Kiparissides (2007) y Villa (2007).

En el caso de la polimerización por adición, las etapas básicas de la reacción son conocidas en forma general, éstas son: iniciación, propagación, terminación y transferencia. Sin embargo, en muchos casos, el mecanismo detallado es complejo y no completamente entendido. Por consiguiente, los modelos recurren a: (i) factores empíricos de ajuste; o (ii) postulados que representan solamente aproximaciones tentativas del mecanismo. El primer recurso es muy frecuente en aplicaciones prácticas inmediatas y de gran importancia en ambientes industriales. El segundo caso está relacionado con el conocimiento de la cinética de reacción y es el motivo del presente trabajo, por lo que solo nos referiremos a este aspecto de los modelos.

Adicionalmente, los modelos que se han elaborado consideran frecuentemente especies químicas difíciles de cuantificar experimentalmente, por ejemplo: complejos que se forman (Rzaev, 2000; Liu et al., 2003), agregados moleculares (Niu et al., 2004; Smid et al., 2006; Baskaran y Müller, 2007), radicales libres de diferentes tamaños (Rivero, 2004; 2006; Achilias, 2007; Nikitin y Hutchinson, 2007), entre otros. En estos casos la validez del modelo solo puede establecerse a través del efecto de dichas entidades en las variables medibles como son: velocidad de reacción y características moleculares del producto polimérico. La simulación matemática ofrece una vía para estudiar esta clase de relaciones. Es posible modificar la concentración de estas entidades, cambiar su reactividad o modificar en general un mecanismo y analizar mediante simulación el efecto sobre las variables medibles. La comparación de los resultados de la simulación con los datos experimentales permite descartar o reforzar ciertos postulados sobre el mecanismo. Es conveniente contrastar contra diferentes clases de datos con el fin de poder discriminar entre diferentes factores que ocasionan una modificación de la variable medible.

El objetivo del presente trabajo es plantear el uso de la simulación matemática no solo para reproducir el comportamiento de sistemas de polimerización sino como una manera de obtener un mayor conocimiento de la cinética de la reacción. Con el propósito de mostrar la forma de abordar y manejar las peculiaridades de diferentes sistemas de polimerización, en este trabajo se describen 
dos ejemplos en los cuales mediante un análisis de los modelos se obtienen conclusiones acerca del mecanismo de reacción.

\section{DESARROLLO}

Partiendo de la estructura básica de un modelo $\mathbf{y}(\mathbf{x}, \mathbf{p})$, donde $\mathbf{x}$ representa las variables de entrada, y las variables de salida y $\mathbf{p}$ los parámetros del modelo, se elaboró un programa computacional. El programa consta de diferentes módulos para facilitar la captura de los datos de entrada, la optimización de parámetros del modelo, la organización, manejo y almacenamiento de datos y la representación gráfica de las variables de salida, conjuntamente con los datos experimentales. La parte esencial del programa es el módulo que contiene los modelos. Este módulo se puede ampliar para incluir todos los modelos que se deseen o sustituir con nuevos módulos para abordar problemas de diferente índole. Adicionalmente, el programa se complementó con un módulo de simulación de reactores por lotes, el cual permite calcular una serie de variables $\mathbf{Y}(\mathrm{t})$ mediante un modelo $\mathbf{y}(\mathbf{x}, \mathbf{p}) \mathbf{a}$ partir de un conjunto de condiciones iniciales.

Las variables de salida del modelo o los resultados de la simulación deben ser contrastados con datos experimentales para establecer la validez del modelo. Dependiendo del sistema de polimerización en particular y los datos experimentales disponibles, puede ser conveniente probar diferentes modelos y sus variantes y comparar los resultados calculados con datos experimentales de diferente clase. Para aclarar el procedimiento se describen a continuación dos ejemplos. El primero trata sobre el problema de desarrollar un modelo de un sistema complejo a partir de sistemas más simples, donde además existe una controversia en cuanto al modelo que mejor describe la cinética de polimerización. El segundo ejemplo se enfoca a tratar de explicar un aspecto de la polimerización aniónica que no puede ser descrito con el modelo actualmente aceptado.

\section{Copolimerización de monómeros que forman complejos del tipo donador aceptador de electrones}

En estos sistemas existen diferentes modelos de la etapa de propagación, los más populares son: modelo terminal, modelo penúltimo, modelo de participación del complejo y modelo de disociación del complejo. El aspecto central es que existe una controversia en cuanto a la posible participación de los complejos en la reacción de propagación o bien el comportamiento peculiar de estos sistemas se deba a simples efectos penúltimos (Rzaev, 2000; Liu et al., 2003). También se han propuesto modelos que consideran ambas posibilidades, es decir el modelo de participación del complejo pero con efectos penúltimos; y de manera más general se ha propuesto una combinación de este modelo con el de disociación del complejo, en el llamado por los autores, modelo omega (Georgiev y Georgieva, 1999). En cuanto a las etapas de iniciación, terminación y transferencia, se pueden plantear diferentes alternativas para modelarlas. Por ejemplo, la etapa de terminación puede o no estar controlada por fenómenos de difusión y depender del tamaño de los radicales, para lo cual existen diferentes modelos (Achilias, 2007; Nikitin y Hutchinson, 2007).

Se analizaron con cada uno de los modelos de propagación mencionados, los datos experimentales de composición de copolímeros del sistema anhídrido maleico-acetato de vinilo. Se determinaron las relaciones de reactividad en cada modelo y se compararon las bondades de ajuste de cada modelo. Adicionalmente, se analizaron los modelos comparando con los datos de velocidad de polimerización reportados por Fujimori y Brown (1986). Se probaron los modelos de propagación mencionados con diferentes modelos de terminación y se incluyeron reacciones de transferencia con y sin retardo, así como todas las combinaciones de estos modelos.

\section{Polimerización aniónica de estireno iniciada con alquilos de litio en disolventes alifáticos}

El esquema general de la polimerización aniónica se ha descrito desde tiempo atrás (Szwarc y Van Beylen, 1993) y revisado recientemente (Smid et al., 2006; Baskaran y Müller, 2007). En ausencia de impurezas que reaccionen con los compuestos de litio, la polimerización se lleva a cabo sin reacciones de terminación o transferencia. Los compuestos de litio, iniciador y polímero, forman agregados moleculares en disolventes no polares y se considera que se establece un equilibrio entre especies libres y agregadas (ecuaciones 1 y 3, para iniciador y polímero respectivamente). Se 
considera que dichos agregados moleculares son inertes y por consecuencia las reacciones de iniciación (ecuación 2) y propagación (ecuación 4) se llevan a cabo a través de la pequeña fracción de compuestos disgregados. En el caso particular de la polimerización de estireno iniciada con secbutilo de litio, el esquema de reacción es el siguiente:

$\mathrm{I}_{4} \stackrel{\mathrm{K}_{\mathrm{d}}}{\longleftrightarrow} 4 \mathrm{I}$

$\mathrm{I}+\mathrm{M} \stackrel{\mathrm{k}_{\mathrm{i}}}{\longrightarrow} \mathrm{P}_{1}$

$\overline{\mathrm{P}}_{\mathrm{n}} \mathrm{P}_{\mathrm{m}} \stackrel{\mathrm{K}_{\mathrm{p}}}{\longrightarrow} \mathrm{P}_{\mathrm{n}}+\mathrm{P}_{\mathrm{m}}$

$\mathrm{P}_{\mathrm{n}}+\mathrm{M} \stackrel{\mathrm{k}}{\longrightarrow} \mathrm{P}_{\mathrm{n}+1}$

Bajo este esquema de reacción la velocidad de iniciación, considerando que $\mathrm{I}<<\mathrm{I}_{4}$, es:

$\mathrm{R}_{\mathrm{i}}=\mathrm{k}_{\mathrm{i}}\left(\mathrm{K}_{\mathrm{d}} / 6\right)^{1 / 4} \mathrm{I}_{\mathrm{T}}^{1 / 4} \mathrm{M}=\mathrm{k}_{\mathrm{i}}^{\prime} \mathrm{I}_{\mathrm{T}}^{1 / 4} \mathrm{M}$

Donde $\mathrm{I}_{\mathrm{T}}$ es la concentración total de iniciador (agregado y disgregado) y $\mathrm{M}$ es la concentración de monómero. La velocidad de propagación, considerando la concentración de polímero libre despreciable frente a la concentración de polímero que forma parte de los agregados, es:

$R_{p}=k\left(K_{p} / 2\right)^{1 / 2} P_{T}^{1 / 2} M=k_{p}^{\prime} P_{T}{ }^{1 / 2} M$

Donde $\mathrm{P}_{\mathrm{T}}$ es la concentración total de polímero "vivo". Si la velocidad de iniciación es al menos igual a la velocidad de propagación, la distribución de pesos moleculares (MWD) conforma una distribución de Poisson. Cualquier desviación de la distribución observada se ha atribuido básicamente a fallas experimentales o a una iniciación lenta (Johnson et al., 1999).

El anterior esquema es aceptado generalmente y describe cercanamente la cinética de polimerización de estireno y algunos dienos en solventes aromáticos como benceno. Las distribuciones de pesos moleculares obtenidas en estos casos se aproximan a la distribución de Poisson con bajos valores de polidispersidad (Johnson, 1999). Sin embargo, cuando el solvente es del tipo alifático, por ejemplo ciclohexano, el comportamiento de la reacción cambia. La reacción de iniciación exhibe un comportamiento autocatalítico, acelerándose la reacción conforme se comienza a consumir el iniciador (Bywater y Worsfold, 1967; Niu et al., 2004). Asimismo, la MWD se ensancha y los índices de polidispersidad aumentan. Este comportamiento no puede ser simulado con el modelo descrito.

Para explicar este comportamiento se recurrió a un análisis por simulación. Se elaboró un modelo, en el cual todas las especies orgánicas de litio, estén o no agregadas, pueden reaccionar con monómero en reacciones de iniciación o propagación. De estas especies orgánicas de litio es importante resaltar que se incluyeron los agregados cruzados iniciador-polímero. Dichos agregados son conocidos y son fácilmente detectados, pero no habían sido incluidos anteriormente en el esquema cinético, no obstante que su sola presencia modifica los equilibrios de disgregación y por lo tanto las concentraciones de las demás especies orgánicas de litio. El equilibrio entre los agregados cruzados y las especies libres se puede plantear como: 
$\overline{\mathrm{P}_{\mathrm{n}} \mathrm{I}} \stackrel{\mathrm{K}_{\mathrm{c}}}{\longleftrightarrow} \mathrm{P}_{\mathrm{n}}+\mathrm{I}$

Las ecuaciones de balance de polímero total de cada tamaño se muestran a continuación. El primer término del lado derecho de la ecuación 8 representa la rapidez de iniciación correspondiente a las reacciones del monómero con el iniciador libre, el iniciador contenido en los agregados de iniciador y el iniciador de los agregados cruzados. Los demás términos del lado derecho de las ecuaciones 8 y 9 corresponden a la rapidez de propagación a través del polímero libre, el polímero de los agregados de polímero y el polímero de los agregados cruzados.

$$
\begin{aligned}
& \frac{d P_{1 T}}{d t}=k_{i} M\left[I+r_{i s} l_{4}+r_{i c} \sum_{m=1}^{\infty} \overline{P_{m} l}\right]-k M\left[P_{1}+r_{s} \sum_{m=1}^{\infty}\left(1+\delta_{1, m}\right) \overline{P_{1} P_{m}}+r_{c} \overline{P_{1} l}\right] \\
& \left.\frac{d P_{n T}}{d t}=k M\left\{\left[P_{n-1}-P_{n}\right]+r_{s}\left[\sum_{m=1}^{\infty}\left(1+\delta_{n-1, m}\right) \overline{P_{n-1} P_{m}}-\sum_{m=1}^{\infty}\left(1+\delta_{n, m}\right) \overline{P_{n} P_{m}}\right]+r_{c}\left[\overline{P_{n-1} l}-\overline{P_{n}}\right]\right]\right\}
\end{aligned}
$$

donde $P_{n T}$ es la concentración total de polímero de tamaño $n, t$ es el tiempo, $r_{\text {is }}$ es la razón entre las constantes $k_{i s} / k_{i}, k_{\text {is }}$ es la constante de iniciación de agregados de iniciador, $r_{\text {ic }}$ es la relación $k_{\text {ic }} / k_{i}$, $k_{\text {ic }}$ es la constante de iniciación de los agregados cruzados, $r_{s}$ es la relación $k_{s} / k, k_{s}$ es la constante de propagación de los agregados de polímero, $r_{c}$ es la relación $k_{c} / k, k_{c}$ es la constante de propagación de los agregados cruzados y $\delta_{n, m}=1$ si $n=m$ de otra manera es cero.

El conjunto infinito de ecuaciones de balance se resolvió por el método de los momentos. Se llevaron a cabo simulaciones y se compararon los resultados con datos experimentales de conversión de monómero, conversión de iniciador y evolución de los pesos moleculares promedio. Se analizó el efecto de cada reacción planteada sobre los resultados de la simulación. El estudio experimental y los resultados completos se describirán en un artículo posterior. En este trabajo solo se presentan los avances del estudio con el fin de mostrar las posibilidades del análisis de modelos mediante simulación.

\section{RESULTADOS Y DISCUSIÓN}

Para el caso de copolimerización de monómeros que forman complejos, la figura 1 muestra los resultados de composición obtenidos mediante el modelo de participación del complejo y los datos experimentales de Brown et al. (1989) del sistema acetato de vinilo-anhídrido maleico. En esta figura $F_{M A}$ y $f_{M A}$ corresponden a la fracción de anhídrido maleico en el copolímero y en la mezcla de monómeros respectivamente. Todos los modelos probados produjeron un comportamiento idéntico, excepto el modelo terminal que mostró ligeras desviaciones. Estos resultados indican que con datos de composición de copolímeros no es posible hacer una clara discriminación entre los diferentes modelos. Sin embargo, al analizar conjuntamente los datos de composición con los de velocidad de copolimerización reportados por Fujimori y Brown (1986) del mismo sistema, los resultados mostraron que el modelo de participación del complejo combinado con terminación controlada por difusión es una base adecuada para explicar los datos. Esto demuestra la importancia de llevar a cabo la comparación simultánea con diversas clases de datos experimentales.

El análisis de otros sistemas de polimerización, como los formados por cloruro de vinilo-anhídrido maleico y acetato de vinilo-cloruro de vinilo, permitió plantear un modelo general de polimerización de 3 monómeros que forman complejos (Rivero y Etchechury, 2001). El modelo permite calcular composición del terpolímero, velocidad de polimerización y pesos moleculares promedio. La figura 2 
muestra la predicción del modelo de la composición de anhídrido maleico en el terpolímero para la polimerización de cloruro de vinilo, acetato de vinilo y anhídrido maleico. Las relaciones de reactividad utilizadas en la construcción de las figuras 1 y 2, y las predicciones de pesos moleculares promedio pueden ser consultadas en la referencia mencionada.

Por otro lado, en el caso de la polimerización aniónica de estireno, la capacidad del modelo para representar la aceleración de la reacción de iniciación es notable, como se muestra en la figura 3. Esto se logra, de acuerdo al modelo, por la participación de los agregados cruzados de iniciadorpolímero en las reacciones de iniciación. La explicación se encuentra en el comportamiento de los agregados cruzados, los cuales se forman una vez que la reacción de iniciación produce polímero y aumenta su concentración conforme aumenta la concentración del polímero formado. Al continuar el proceso de iniciación, el iniciador se consume paulatinamente de tal manera que la concentración de los agregados cruzados comienza a disminuir. Por consiguiente, el perfil de concentración de los agregados cruzados muestra un ascenso, llega a un máximo y después disminuye, como se puede observar en la figura 3. Este perfil corresponde muy cercanamente al perfil de velocidad de iniciación.

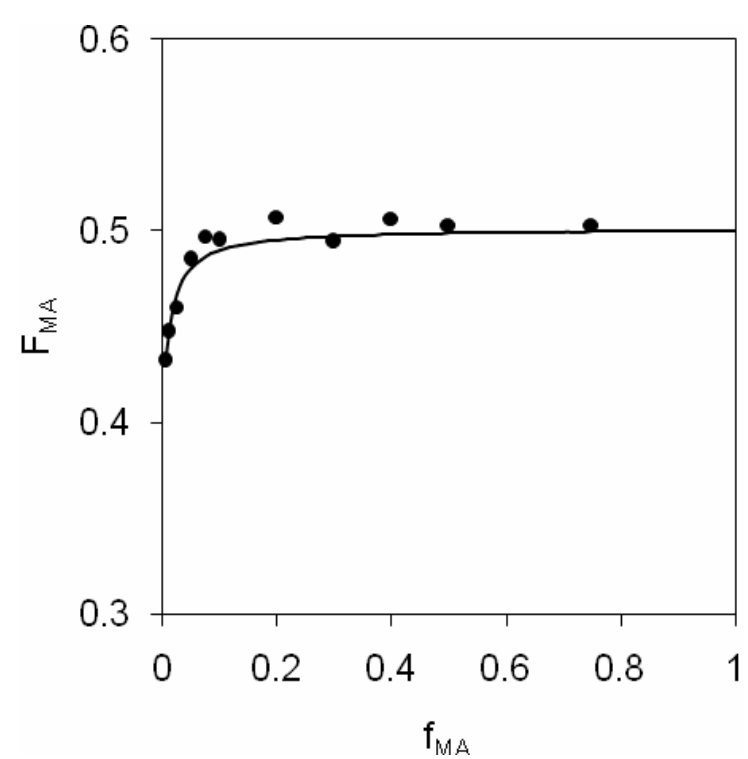

Fig. 1: Modelo de participación del complejo y datos de Brown et al. (1989). Copolímerización de acetato de vinilo y anhídrido maleico

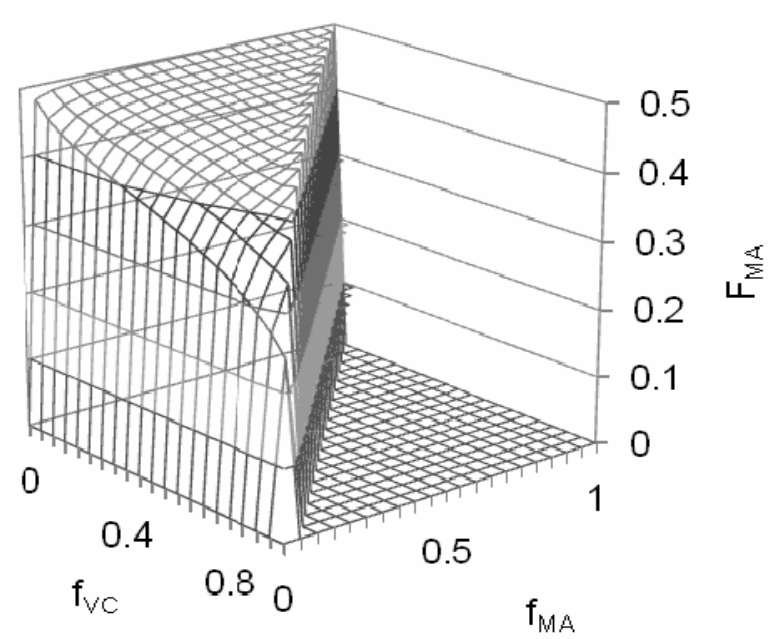

Fig. 2: Predicción de la composición de anhídrido maleico en terpolímeros de cloruro de vinilo, acetato de vinilo y anhídrido maleico

De acuerdo al esquema de reacciones planteado y a las consecuentes ecuaciones de balance (ecuaciones 8 y 9), la única posibilidad para que el perfil de concentración del agregado cruzado concuerde con el de velocidad de iniciación es que la reactividad de los agregados cruzados no sea cero. Por supuesto, en un sentido riguroso, la coincidencia de ambos perfiles no significa necesariamente la participación de los agregados cruzados en las reacciones de iniciación; se requiere de evidencias experimentales más directas para afirmarlo, por lo que en este trabajo planteamos esta explicación solo como una hipótesis plausible. Sin embargo, se considera que esta hipótesis, de la participación de los agregados cruzados en las reacciones de iniciación, es la explicación más consistente y lógica de los datos experimentales, dado el estado de los conocimientos que se tienen de la reacción. Por supuesto, con el esquema cinético aceptado (es decir mediante la ecuación 5) no es posible representar los perfiles velocidad de iniciación o la curva de conversión del iniciador de la figura 3.

Los parámetros del modelo utilizados para la simulación mostrada en la figura 3 se obtuvieron a partir de: (i) determinación experimental de la constante de propagación aparente $k_{p}^{\prime}$ definida mediante la ecuación 6; (ii) suposición de $r_{s}=0$, con base en el valor bien establecido del orden de reacción $1 / 2$ con respecto a la concentración de polímero "vivo" en la propagación de estireno; (iii) 
suposición de $r_{c}=0$, considerando que las reacciones de propagación de polímero contenido en los agregados cruzados tiene un efecto menor en la conversión de monómero y en los pesos moleculares; (iv) suposición de la constante de agregación de polímero $\mathrm{K}_{\mathrm{p}}$ definida en la ecuación 3; y (v) ajuste del modelo a los datos experimentales mediante una optimización multivariable utilizando el método del gradiente conjugado y una aproximación cuadrática a las funciones de una variable generadas en cada dirección de búsqueda. Los parámetros obtenidos por este procedimiento son: $\mathrm{k}_{\mathrm{i}}$ $=1.53 \mathrm{~L} \mathrm{~mol}^{-1} \mathrm{~s}^{-1}, \mathrm{~K}_{\mathrm{d}}=10^{-20} \mathrm{~mol}^{3} \mathrm{~L}^{-3}, \mathrm{r}_{\text {is }}=0, \mathrm{r}_{\mathrm{ic}}=0.0497, \mathrm{k}=75.3 \mathrm{~L} \mathrm{~mol}^{-1} \mathrm{~s}^{-1}, \mathrm{~K}_{\mathrm{p}}=10^{-7} \mathrm{~mol} \mathrm{~L}^{-1}, \mathrm{r}_{\mathrm{s}}=0$, $r_{c}=0, K_{c}=9.6^{\star} 10^{-10} \mathrm{~mol} \mathrm{~L}^{-1}$

Los valores de estos parámetros para las condiciones de la reacción deben tomarse con reserva. El procedimiento de optimización multivariable utilizado produce valores de los parámetros que en conjunto son numéricamente consistentes con los datos experimentales pero individualmente no garantizan que realmente representen cada etapa del mecanismo. No obstante, se recurre a este procedimiento matemático porque desafortunadamente las constantes absolutas de iniciación y propagación no se conocen y las constantes de los equilibrios de disociación de agregados no se encuentran disponibles en la literatura.

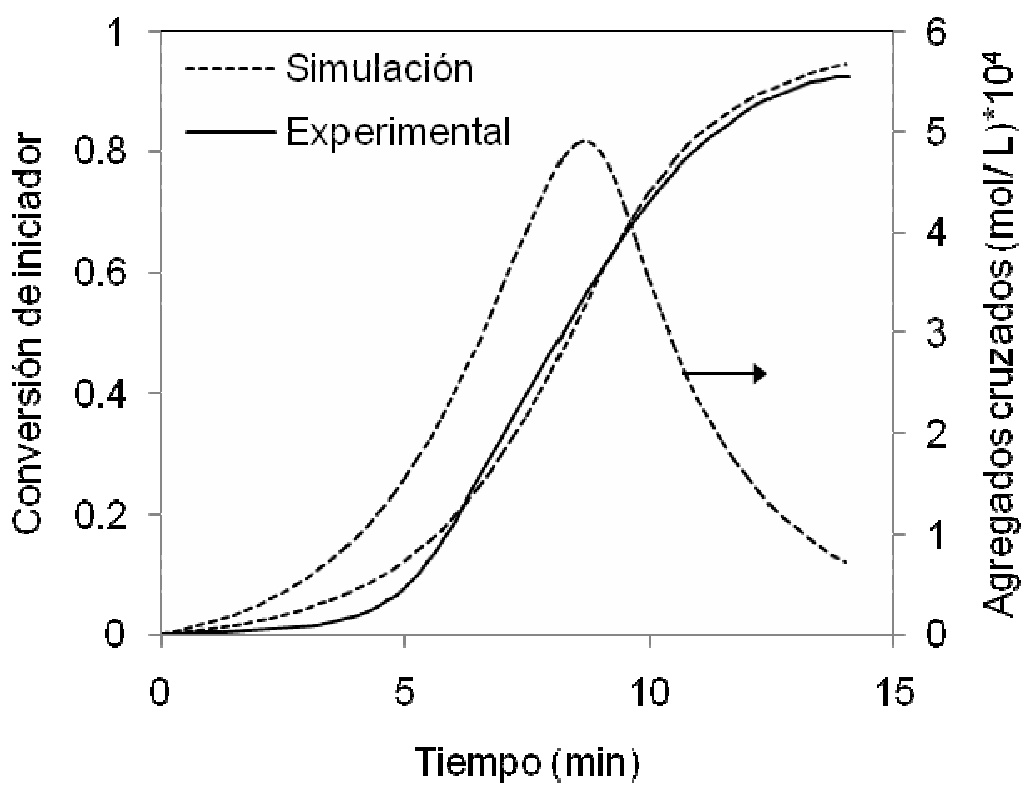

Fig. 3: Simulación de la conversión de iniciador y concentración de agregados cruzados de iniciador-polímero y comparación con la curva reportada por Bywater y Worsfold (1967) para la conversión de iniciador en la polimerización de estireno con sec-butilo de litio en cicloehexano a $40^{\circ} \mathrm{C}$

La figura 4 muestra el progreso de una polimerización de estireno usando n-butilo de litio como iniciador y ciclohexano como solvente. Las condiciones de reacción fueron: 0.001125 y $0.571 \mathrm{~mol} \mathrm{~L}^{-1}$ para la concentración de n-butilo de litio y estireno, respectivamente y $57.9^{\circ} \mathrm{C}$. Se observa en la figura la sección lineal de los datos experimentales que corresponden a la etapa de propagación, una vez concluida la iniciación. La pendiente de la línea corresponde a la constante de propagación aparente $k_{p}^{\prime}$, de acuerdo a la ecuación 6 . La primera parte de la reacción de polimerización, de 0 a 5 minutos aproximadamente, corresponde a una combinación de iniciación y propagación.

El modelo planteado en este trabajo permite simular además la evolución de pesos moleculares promedio $M_{n}, M_{w}$ y $M_{z}$ durante la reacción de polimerización. La figura 5 muestra los resultados de la simulación y los datos experimentales de pesos moleculares promedio para la reacción de polimerización de estireno a $61.3^{\circ} \mathrm{C}$ con sec-butilo de litio como iniciador. Las concentraciones de iniciador y monómero son 0.001143 y $0.592 \mathrm{~mol} \mathrm{~L}^{-1}$, respectivamente. En este caso los parámetros 
cinéticos se obtuvieron por el mismo procedimiento explicado anteriormente, excepto que se supuso $r_{i s}$ igual al valor previamente determinado en el ajuste de los datos de conversión de iniciador. Los valores de los parámetros utilizados en la simulación, son: $\mathrm{k}_{\mathrm{i}}=1.70 \mathrm{~L} \mathrm{~mol}^{-1} \mathrm{~s}^{-1}, \mathrm{~K}_{\mathrm{d}}=10^{-23} \mathrm{~mol}^{3} \mathrm{~L}^{-3}, \mathrm{r}_{\text {is }}=$ $0, r_{\text {ic }}=0.0105, \mathrm{k}=432.0 \mathrm{~L} \mathrm{~mol}^{-1} \mathrm{~s}^{-1}, \mathrm{~K}_{\mathrm{p}}=10^{-7} \mathrm{~mol} \mathrm{~L}^{-1}, \mathrm{r}_{\mathrm{s}}=0, \mathrm{r}_{\mathrm{c}}=0, \mathrm{~K}_{\mathrm{c}}=3.1 * 10^{-9} \mathrm{~mol} \mathrm{~L}^{-1}$.

La comparación entre la simulación y los datos experimentales de la figura 5 permite reforzar la hipótesis de la participación de los agregados cruzados en las reacciones de iniciación y la validez del modelo desarrollado.

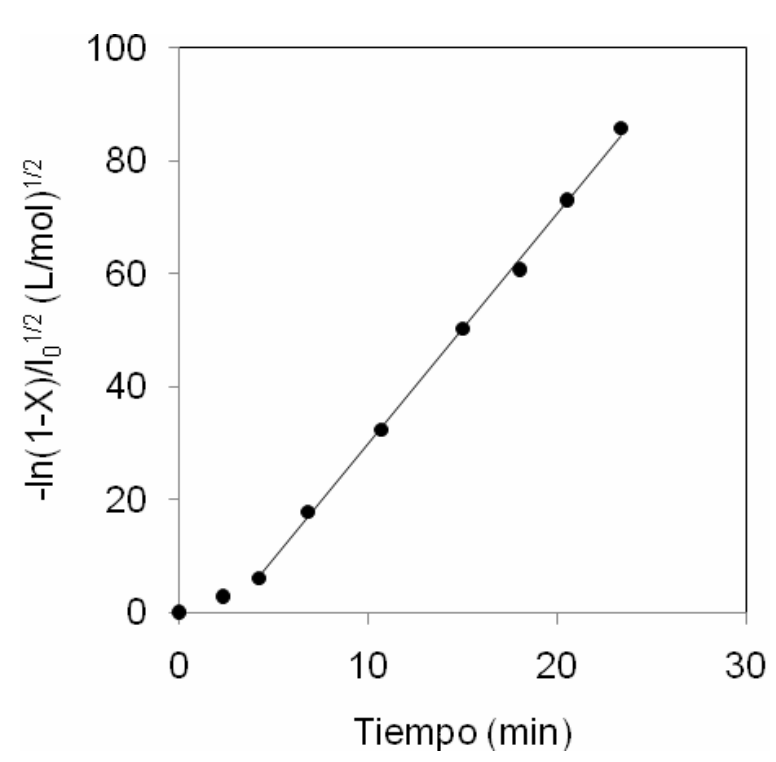

Fig. 4: Conversión de monómero en la reacción de polimerización de estireno con n-butilo de litio a $57.9^{\circ} \mathrm{C}$

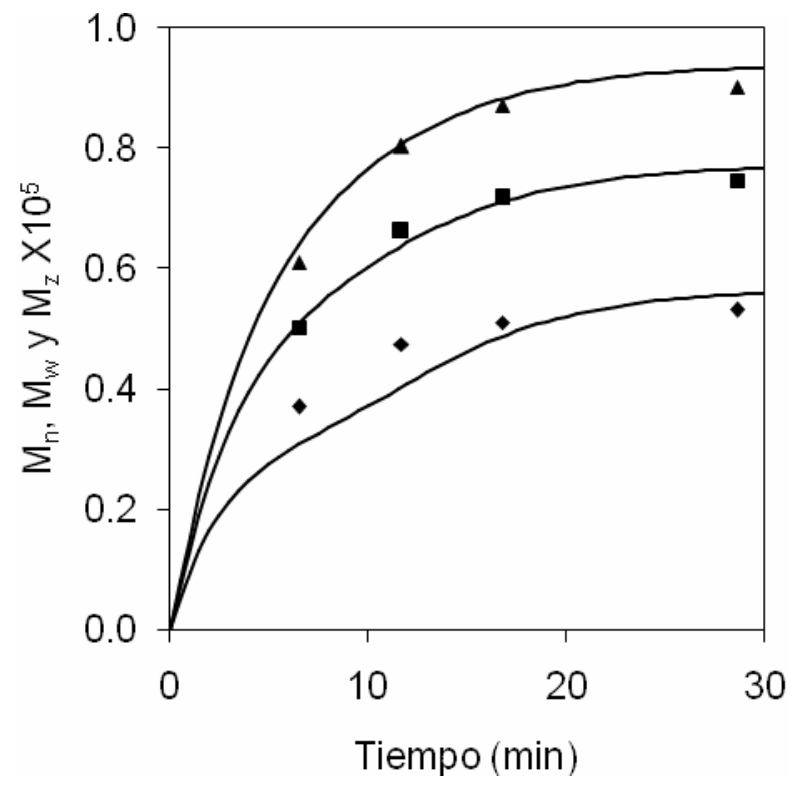

Fig. 5: Evolución de los pesos moleculares promedio simulados y experimentales en la polimerización de estireno con sec-butilo de litio

\section{CONCLUSIONES}

Los modelos matemáticos de cinética de polimerización establecen una serie de relaciones entre variable; algunas de de estas relaciones no se conocen o no se entienden completamente con los conocimientos actuales. En este trabajo mostramos la utilidad de la simulación matemática para analizar modelos de cinética de polimerización y contribuir al conocimiento del mecanismo de reacción. Los dos ejemplos descritos, ilustran el procedimiento de análisis y muestran los alcances de éste. En el caso de la polimerización de monómeros que forman complejos fue posible construir un modelo general de terpolimerización a partir del análisis de sistemas binarios más simples. En un segundo ejemplo, de polimerización aniónica de estireno el análisis condujo al planteamiento de un aspecto novedoso del mecanismo de reacción que no se había reportado antes: la participación de los agregados cruzados de iniciador-polímero en reacciones de iniciación. En ambos ejemplos la simulación matemática mostró ser una herramienta de análisis de gran valor que permite plantear nuevos conocimientos o hipótesis sobre cinética de polimerización.

\section{NOMENCLATURA}

I Iniciador o su concentración.

$\mathrm{I}_{0} \quad$ Concentración de iniciador a $\mathrm{t}=0$.

$\mathrm{I}_{4} \quad$ Agregado de iniciador.

$\mathrm{I}_{\mathrm{T}} \quad$ Iniciador total o su concentración.

$\mathrm{F}_{\mathrm{MA}} \quad$ Fracción de anhídrido maleico en el copolímero.

$\mathrm{f}_{\mathrm{MA}} \quad$ Fracción de anhídrido maleico en la mezcla de monómeros.

$\mathrm{f}_{\mathrm{vc}} \quad$ Fracción de cloruro de vinilo en la mezcla de monómeros. 
k Coeficiente de velocidad de propagación de polímero disociado.

$\mathrm{k}_{\mathrm{i}}^{\prime} \quad$ Coeficiente aparente de iniciación (ecuación 5).

$\mathrm{k}_{\mathrm{p}}^{\prime} \quad$ Coeficiente aparente de velocidad de propagación (ecuación 6).

$\mathrm{K}_{\mathrm{c}} \quad$ Constante de equilibrio de agregados cruzados (ecuación 7).

$\mathrm{k}_{\mathrm{c}}$

$\mathrm{K}_{\mathrm{d}}$

Coeficiente de velocidad de propagación de agregados cruzados de iniciador-polímero.

Constante de equilibrio de los agregados de iniciador (ecuación 1).

$k_{i} \quad$ Coeficiente de velocidad de iniciación de iniciador disociado.

$\mathrm{k}_{\text {ic }} \quad$ Coeficiente de velocidad de iniciación de agregados cruzados de iniciador-polímero.

$\mathrm{k}_{\mathrm{is}} \quad$ Coeficiente de velocidad de iniciación de agregados de iniciador.

$\mathrm{K}_{\mathrm{p}} \quad$ Constante de equilibrio de los agregados de polímero (ecuación 3).

$\mathrm{k}_{\mathrm{s}} \quad$ Coeficiente de velocidad de propagación de agregados de polímero.

M Monómero o su concentración.

$\mathrm{M}_{\mathrm{n}} \quad$ Peso molecular promedio en número.

$\mathrm{M}_{\mathrm{w}} \quad$ Peso molecular promedio en peso.

$\mathrm{M}_{\mathrm{z}} \quad$ Peso molecular promedio z.

p Parámetros del modelo.

$\mathrm{P}_{\mathrm{n}} \quad$ Polímero de longitud $\mathrm{n}$, o su concentración.

$\overline{\mathrm{P}_{\mathrm{n}} \mathrm{I}} \quad$ Agregados cruzados de iniciador y polímero de longitud $\mathrm{n}$, o su concentración.

$\overline{\mathrm{P}}_{\mathrm{n}} \mathrm{P}_{\mathrm{m}} \quad$ Agregados de polímero de longitud $\mathrm{n}$ y polímero de longitud $\mathrm{m}$, o su concentración.

$\mathrm{P}_{\mathrm{nT}} \quad$ Concentración total de polímero de longitud $\mathrm{n}$.

$\mathrm{P}_{\mathrm{T}} \quad$ Concentración total de polímero.

$r_{c} \quad$ Relación de reactividad $k_{c} / k$.

$\mathrm{R}_{\mathrm{i}} \quad$ Velocidad de iniciación.

$r_{\text {ic }} \quad$ Relación de reactividad $k_{i c} / k_{i}$.

$r_{\text {is }} \quad$ Relación de reactividad $k_{i s} / k_{i}$.

$\mathrm{R}_{\mathrm{p}} \quad$ Velocidad de propagación.

$\mathrm{r}_{\mathrm{s}} \quad$ Relación de reactividad $\mathrm{k}_{\mathrm{s}} / \mathrm{k}$.

$\mathrm{t} \quad$ Tiempo.

X Conversión de monómero.

$x \quad$ Conjunto de variables de entrada de un modelo.

y Conjunto de variables de salida de un modelo.

Y Conjunto de variables simuladas.

$\delta_{\mathrm{n}, \mathrm{m}} \quad$ Delta de Kronecker.

\section{REFERENCIAS}

Achilias, D.S.; A Review of Modeling of Diffusion Controlled Polymerization Reactions, Macromol. Theory Simul.: 16(4), 319-347 (2007).

Baskaran, D. y A.H.E. Müller; Anionic vinyl polymerization-50 years after Michael Szwarc, Progr. Polym. Sci.: 32, 173-219 (2007).

Brown, A.S., K. Fujimori y I. Craven; The Mechanism of Copolymerization of Maleic Anhydride with Styrene and with Vinyl Acetate, J. Polym. Sci., Part A, Polym. Chem.: 27, 3315-3325 (1989).

Bywater, S. y D.J. Worsfold; Alkyllithium Anionic Polymerization Initiators in Hydrocarbon Solvents, J. Organometal. Chem.: 10, 1-6 (1967).

Dubé, M.A., J.B.P. Soares, A. Penlidis y A.E. Hamielec; Mathematical Modeling of Multicomponent Chain-Growth Polymerizations in Batch, Semibatch, and Continuous Reactors: A Review, Ind. Eng. Chem. Res.: 36(4), 966-1015 (1997).

Fujimori, K. y A.S. Brown; Overall Rate of Alternating Copolymerization of Vinyl Acetate with Maleic Anhydride in Methyl Ethyl Ketone Relative Reactivity of the Complex and Free Monomers, Polym. Bull.: 15, 223-226 (1986). 
Gao, J. y A. Penlidis; A Comprehensive Simulator/Database Package for Reviewing Free-Radical Homopolymerizations, J. Macromol. Sci. Rev. Macromol. Chem. Phys.: C36(2), 199-404 (1996).

Gao, J. y A. Penlidis; A Comprehensive Simulator/Database Package for bulk/solution Free-Radical Terpolymerizations, Macromol. Chem. Phys.: 201(11), 1176-1184 (2000).

Gao, J. y A. Penlidis; Mathematical Modeling and Computer Simulation/Database for Emulsion Polymerizations, Prog. Polym. Sci.: 27, 403-535 (2002).

Georgiev, G.S. y V.T. Georgieva; Generalized $(\Omega)$ Model of Radical Copolymerization. Part 1. Combined Comppen Model, Polym. Int.: 48, 819-831 (1999).

Johnson, A.F., M.A. Mohsin, Z.G. Meszena y P. Graves-Morris; Predicted and Observed Molecular Weight Distributions and Dispersity Indices in Living Polymerization Processes: Some Comments, J. Macromol. Sci., Rev. Macromol. Chem. Phys.: C39(3), 527-560 (1999).

Krallis, A. y C. Kiparissides; Mathematical Modeling of the Bivariate Molecular Weight -Long Chain Branching Distribution of Highly Branched Polymers. A Population Balance Approach, Chem. Eng. Sci.: 62, 5304-5311 (2007).

Liu, G.D., Y. Zhang, X.W. Qu y L.C Zhang; Kinetic Model of Mechanism of Alternating Radical Copolymerization and its Quantitative Treatment Method, Polym. Int.: 52, 987-992 (2003).

Nikitin, A.N. y R.A. Hutchinson; Determination of the Mode of Free Radical Termination from Pulsed Laser Polymerization Experiments, Macromol. Theory Simul.: 16(1), 29-42 (2007).

Niu, A.Z. y otros nueve autores; A New View of the Anionic Diene Polymerization Mechanism, Macromol. Symp.: 215, 1-15 (2004).

Rivero, P. y E. Etchechury; Modelling the Molecular Weight Distribution in Terpolymerization Systems with Donor-Acceptor Complexes, Computational Theoretical Polymer Science: 11, 1-7 (2001).

Rivero, P.; Calculation Method of Molecular Weight Distribution in Polymerization with Chain-LengthDependent Termination, Journal Polymer Research: 11, 309-315 (2004).

Rivero, P.; Simulación de la Polimerización por Radicales Libres en el Intervalo Completo de Conversión, Información Tecnológica: 17(3), 79-85 (2006).

Rzaev, Z.M.O.; Complex Alternating Copolymerization, Progr. Polym. Sci.: 25, 163-217 (2000).

Smid, J., M. Van Beylen y T.E. Hogen-Esch; Perspectives on the contribution of Michael Szwarc to living polymerization, Progr. Polym. Sci.: 31, 1041-1067 (2006).

Szwarc, M. y M. Van Beylen; lonic polymerization and living polymers, 147-172, Chapman \& Hall, New York, USA (1993).

Villa, C.M.; Reactor Modeling for Polymerization Processes, Ind. Eng. Chem. Res.: 46(18), 58155823 (2007). 